\title{
Well-Posedness, Blow-Up Phenomena, and Asymptotic Profile for a Weakly Dissipative Modified Two-Component Camassa-Holm Equation
}

\author{
Yongsheng $\mathrm{Mi}^{1,2}$ and Chunlai $\mathrm{Mu}^{2}$ \\ ${ }^{1}$ College of Mathematics and Computer Sciences, Yangtze Normal University, Chongqing, Fuling 408100, China \\ ${ }^{2}$ College of Mathematics and Statistics, Chongqing University, Chongqing 400044, China \\ Correspondence should be addressed to Yongsheng Mi; miyongshen@163.com
}

Received 20 March 2013; Revised 27 June 2013; Accepted 3 July 2013

Academic Editor: Michael Meylan

Copyright (C) 2013 Y. Mi and C. Mu. This is an open access article distributed under the Creative Commons Attribution License, which permits unrestricted use, distribution, and reproduction in any medium, provided the original work is properly cited.

We study the Cauchy problem of a weakly dissipative modified two-component Camassa-Holm equation. We firstly establish the local well-posedness result. Then we present a precise blow-up scenario. Moreover, we obtain several blow-up results and the blowup rate of strong solutions. Finally, we consider the asymptotic behavior of solutions.

\section{Introduction}

In this paper, we consider the Cauchy problem of the following weakly dissipative modified two-component CamassaHolm system:

$$
\begin{gathered}
m_{t}+u m_{x}+2 m u_{x}+\rho \bar{\rho}_{x}+\lambda m=0, \\
t>0, x \in \mathbb{R}, \\
\rho_{t}+(\rho u)_{x}+\lambda \rho=0, \quad t>0, x \in \mathbb{R}, \\
m(0, x)=m_{0}(x), \quad x \in \mathbb{R}, \\
\rho(0, x)=\rho_{0}(x), \quad x \in \mathbb{R},
\end{gathered}
$$

where $m=\left(1-\partial_{x}^{2}\right) u, \rho=\left(1-\partial_{x}^{2}\right)\left(\bar{\rho}-\bar{\rho}_{0}\right)$, and $\lambda$ is a nonnegative dissipative parameter.

The Camassa-Holm equation [1] has been recently extended to a two-component integrable system (CH2)

$$
\begin{gathered}
m_{t}+u m_{x}+2 m u_{x}=\rho \rho_{x}, \quad t>0, x \in \mathbb{R} \\
\rho_{t}+(\rho u)_{x}=0, \quad t>0, x \in \mathbb{R}
\end{gathered}
$$

with $m=u-u_{x x}$, which is a model for wave motion on shallow water, where $u(t, x)$ describes the horizontal velocity of the fluid and $\rho(t, x)$ is in connection with the horizontal deviation of the surface from equilibrium, all measured in dimensionless units. Moreover, $u$ and $\rho$ satisfy the boundary conditions: $u \rightarrow 0$ and $\rho \rightarrow 1$ as $|x| \rightarrow \infty$. The system can be identified with the first negative flow of the AKNS hierarchy and possesses the interesting peakon and multikink solutions [2]. Moreover, it is connected with the timedependent Schrödinger spectral problem [2]. Popowicz [3] observes that the system is related to the bosonic sector of an $N=2$ supersymmetric extension of the classical Camassa-Holm equation. Equation (2) with $\rho \equiv 0$ becomes the Camassa-Holm equation, which has global conservative solutions [4] and dissipative solutions [5]. For other methods to handle the problems relating to various dynamic properties of the Camassa-Holm equation and other shallow water equations, the reader is referred to [6-8] and the references therein.

Since the system was derived physically by Constantin and Ivanov [9] in the context of shallow water theory (also by Chen et al. in [2] and Falqui in [10]), many researchers have paid extensive attention to it. In [11], Escher et al. establish the local well-posedness and present the precise blow-up scenarios and several blow-up results of strong solutions to (2) on the line. In [9], Constantin and Ivanov investigate the global existence and blow-up phenomena of strong solutions of (2) on the line. Later, Guan and Yin [12] obtain a new global existence result for strong solutions to (2) and get several 
blow-up results, which improve the recent results in [9]. Recently, they study the global existence of weak solutions to (2) [13]. In [14], Henry studies the infinite propagation speed for (2). Gui and Liu [15] establish the local well-posedness for (2) in a range of the Besov spaces; they also derive a wavebreaking mechanism for strong solutions. Mustafa [16] gives a simple proof of existence for the smooth travelling waves for (2). Hu and Yin $[17,18]$ study the blow-up phenomena and the global existence of (2) on the circle.

Recently, the $\mathrm{CH} 2$ system was generalized into the following modified two-component Camassa-Holm (MCH2) system:

$$
\begin{gathered}
m_{t}+u m_{x}+2 m u_{x}=-g \rho \bar{\rho}_{x}, \quad t>0, x \in \mathbb{R} \\
\rho_{t}+(\rho u)_{x}=0, \quad t>0, \quad x \in \mathbb{R}
\end{gathered}
$$

where $m=\left(1-\partial_{x}^{2}\right) u, \rho=\left(1-\partial_{x}^{2}\right)\left(\bar{\rho}-\bar{\rho}_{0}\right), u$ denotes the velocity field, $\bar{\rho}_{0}$ is taken to be a constant, and $g$ is the downward constant acceleration of gravity in applications to shallow water waves. This $\mathrm{MCH} 2$ system does admit peaked solutions in the velocity and average density; we refer this to [19] for details. There, the authors analytically identified the steepening mechanism that allows the singular solutions to emerge from smooth spatially confined initial data. They found that wave breaking in the fluid velocity does not imply singularity in the pointwise density $\rho$ at the point of vertical slope. Some other recent works can be found in [20-31]. We find that the $\mathrm{MCH} 2$ system is expressed in terms of an averaged or filtered density $\bar{\rho}$ in analogy to the relation between momentum and velocity by setting $\rho=\left(1-\partial_{x}^{2}\right)(\bar{\rho}-$ $\left.\bar{\rho}_{0}\right)$, but it may not be integrable unlike the $\mathrm{CH} 2$ system. The important point here is that $\mathrm{MCH} 2$ has the following conservation law $\int_{R}\left(u^{2}+u_{x}^{2}+\rho^{2}+\rho_{x}^{2}\right) d x$, which plays a crucial role in the study of (3), noting that, for the $\mathrm{CH} 2$ system, we cannot obtain the conservation of $H^{1}$ norm.

In general, it is quite difficult to avoid energy dissipation mechanisms in the real world. Ghidaglia [32] studies the long-time behavior of solutions to the weakly dissipative $\mathrm{KdV}$ equation as a finite-dimensional dynamical system. Recently, $\mathrm{Hu}$ and Yin [33] study the blow-up and blowup rate of solutions to a weakly dissipative periodic rod equation. In $[34,35], \mathrm{Hu}$ considered global existence and blow-up phenomena for a weakly dissipative two-component Camassa-Holm system on the circle and on the line. However, (1) on the line (nonperiodic case) has not been studied yet. The aim of this paper is to study the blow-up phenomena and asymptotic profile of the strong solutions to (1). We find that asymptotic profile of solutions to the weakly dissipative modified two-component periodic Camassa-Holm system (1) is similar to that of the modified two-component CamassaHolm system (3), such as the local well-posedness and the blow-up scenario. In addition, we also find that the blow-up rate of (1) is not affected by the weakly dissipative term, but the occurrence of blow-up of (1) is affected by the dissipative parameter.

This paper is organized as follows. In Section 2, we present some notations and establish the local well-posedness for system (1) by applying Kato's semigroup approach to nonlinear hyperbolic evolution equations. In Section 3, we prove a precise blow-up scenario result. In Section 4, we present the blow-up results for strong solutions to (1) provided that the initial data satisfy appropriate conditions and we derive a blow-up rate estimate result. Finally, we consider the asymptotic behavior of solutions.

\section{Local Well-Posedness}

We now provide the framework in which we will reformulate system (1). With $m=u-u_{x x}, \rho=\gamma-\gamma_{x x}$, and $\gamma=\bar{\rho}-\bar{\rho}_{0}$, we can rewrite (1) as follows:

$$
\begin{array}{r}
m_{t}+u m_{x}+2 m u_{x}+\rho \gamma_{x}+\lambda y=0, \\
t>0, x \in \mathbb{R}, \\
\rho_{t}+(\rho u)_{x}+\lambda \rho=0, \quad t>0, x \in \mathbb{R}, \\
y(0, x)=u_{0}(x)-u_{0, x x}(x), \quad x \in \mathbb{R}, \\
\rho(0, x)=\gamma_{0}(x)-\gamma_{0, x x}(x), \quad x \in \mathbb{R} .
\end{array}
$$

Note that if $p(x):=(1 / 2) e^{-|x|}, x \in R$, then $\left(1-\partial_{x}^{2}\right)^{-1} f=p * f$ for all $f \in L^{2}(R), p * y=u$, and $p * \rho=\gamma$. Here, we denote by $*$ the convolution. Using this identity, we can rewrite (5) as follows:

$$
\begin{gathered}
u_{t}+u u_{x}=-\partial_{x} p *\left(u^{2}+\frac{1}{2} u_{x}^{2}+\frac{1}{2} \gamma^{2}-\frac{1}{2} \gamma_{x}^{2}\right)-\lambda u, \\
t>0, x \in \mathbb{R}, \\
\gamma_{t}+u \gamma_{x}=-p *\left(\left(u_{x} \gamma_{x}\right)_{x}+u_{x} \gamma\right)-\lambda \gamma, \\
t>0, x \in \mathbb{R}, \\
u(0, x)=u_{0}(x), \quad x \in \mathbb{R}, \\
\gamma(0, x)=\gamma_{0}(x), \quad x \in \mathbb{R},
\end{gathered}
$$

or we can write it in the equivalent form

$$
\begin{gathered}
u_{t}+u u_{x} \\
=-\partial_{x}\left(1-\partial_{x}^{2}\right)^{-1}\left(u^{2}+\frac{1}{2} u_{x}^{2}+\frac{1}{2} \gamma^{2}-\frac{1}{2} \gamma_{x}^{2}\right)-\lambda u, \\
t>0, x \in \mathbb{R}, \\
\gamma_{t}+u \gamma_{x} \\
=-\partial_{x}\left(1-\partial_{x}^{2}\right)^{-1}\left(u_{x} \gamma_{x}\right)-\left(1-\partial_{x}^{2}\right)^{-1} u_{x} \gamma-\lambda \gamma, \\
\quad t>0, x \in \mathbb{R}, \\
u(0, x)=u_{0}(x), \quad x \in \mathbb{R}, \\
\gamma(0, x)=\gamma_{0}(x), \quad x \in \mathbb{R} .
\end{gathered}
$$

The local well-posedness of the Cauchy problem (5) in Sobolev spaces $H^{s}$ with $s>5 / 2$ can be obtained by applying Kato's theorem $[23,36]$. As a result, we have the following well-posedness result. 
Theorem 1. Given $z_{0}=z(x, 0)=\left(u_{0}, \gamma_{0}\right) \in H^{s} \times H^{s}, s>5 / 2$, then there exist a maximal $T=T\left(\left\|z_{0}\right\|_{H^{s} \times H^{s}}\right)>0$ and a unique solution $z=(u, \gamma)$ to (3) (or (6)) such that

$$
\begin{array}{r}
z=\left(\cdot, z_{0}\right) \in C\left([0, T] ; H^{s} \times H^{s}\right) \\
\cap C^{1}\left([0, T] ; H^{s-1} \times H^{s-1}\right) .
\end{array}
$$

Moreover, the solution depends continuously on the initial data; that is, the mapping $u_{0} \rightarrow u\left(\cdot, u_{0}\right): H^{s} \rightarrow C\left([0, T] ; H^{s}(\mathbb{R})\right) \cap$ $C^{1}\left([0, T] ; H^{s-1}(\mathbb{R})\right)$ is continuous.

\section{The Precise Blow-Up Scenario}

In this section, we present the precise blow-up scenarios for solutions to (6).

Theorem 2. Let $z_{0}=(u, \gamma) \in H^{s} \times H^{s}, s>5 / 2$, be given and assume that $T$ is the maximal existence time of the corresponding solution $z=(u, \gamma)$ to (6) with initial data $z_{0}$; if there exists $M>0$ such that

$$
\begin{aligned}
& \left\|u_{x}(t, \cdot)\right\|_{L^{\infty}}+\|\gamma(t, \cdot)\|_{L^{\infty}}+\left\|\gamma_{x}(t, \cdot)\right\|_{L^{\infty}} \\
& \quad \leq M, \quad t \in[0, T),
\end{aligned}
$$

then the $H^{s} \times H^{s}$ norm of $z(t, \cdot)$ does not blow up on $[0, T)$.

The proof of the theorem is similar to the proof of Theorem 3 in [22]; we omit it here.

Consider the following differential equation:

$$
\begin{gathered}
\frac{d q(x, t)}{d t}=u(q(x, t), t), \quad t \in[0, T), \\
q(0, t)=x, \quad x \in \mathbb{R} .
\end{gathered}
$$

By applying the classical results on the theory of ordinary differential equations, we may derive the following properties of the solution $q$ of (9), which are crucial in the proof of global existence and blow-up of solutions.

Lemma 3 (see [23]). Let $u_{0} \in H^{s}, s \geq 5 / 2$, and let $T$ be the maximal existence time of the corresponding solution $u(t, x)$ to (9). Then (9) has a unique solution $q \in C^{1}([0, T) \times \mathbb{R}, \mathbb{R})$. Moreover, the map $q(t, \cdot)$ is an increasing diffeomorphism of $\mathbb{R}$ with

$$
\begin{aligned}
& q_{x}(x, t)=\exp \left(\int_{0}^{t} u_{x}(q(x, s), s) d s\right)>0, \\
& q_{x}(x, 0)=1, \quad x \in R, 0 \leq t<T .
\end{aligned}
$$

Lemma 4. Let $z_{0}=\left(u_{0}, \gamma_{0}\right) \in H^{s} \times H^{s}$ with $s>5 / 2$, and let $T>0$ be the maximal existence time of the corresponding solution $z \in(u, \gamma)$ to (5). Then, one has

$$
\rho(t, q(x, t)) q_{x}=\rho_{0} e^{-\lambda t}, \quad \forall(t, x) \in[0, T) \times R .
$$

Moreover, if there exists $M_{1}>0$, such that $u_{x}(t, x) \geq-M_{1}$ for all $(t, x) \in[0, T) \times R$, then

$$
\begin{aligned}
\|\rho(t, \cdot)\|_{L^{\infty}} & =\|\rho(t, q(t, x))\|_{L^{\infty}} \\
& \leq e^{M_{1} T}\left\|\rho_{0}(\cdot)\right\|_{L^{\infty}}, \quad \forall t \in[0, T) .
\end{aligned}
$$

Proof. Differentiating the left-hand side of (6) with respect to $t$, in view of (9) and the second equation of (5), we have

$$
\frac{d}{d t}\left(\rho(t, q(x, t)) q_{x}\right)=\rho_{t} q_{x}+\rho_{x} q_{t} q_{x}+\rho q_{x t}=-\lambda \rho q_{x} .
$$

Solving the equation, we get (11).

By Lemma 3, in view of (11) and the assumption of the lemma, we obtain

$$
\begin{aligned}
\|\rho(t, \cdot)\|_{L^{\infty}} & =\|\rho(t, q(t, \cdot))\|_{\infty}=\left\|e^{-\lambda t-\int_{0}^{t} u_{x}(s,) d x} \rho_{0}(\cdot)\right\|_{L^{\infty}} \\
& \leq e^{M_{1} T}\|\rho(\cdot)\|_{L^{\infty}}, \quad \forall t \in[0, T) .
\end{aligned}
$$

The following result is proved only with regard to $r=3$, since we can obtain the same conclusion for the general case $r>5 / 2$ by using Theorem 1 and a simple density argument.

We now present a precise blow-up scenario for strong solutions to (5).

Theorem 5. Let $y_{0}=\left(u_{0}, \gamma_{0}\right) \in H^{s} \times H^{s}, s>5 / 2$, and let $T$ be the maximal existence of the corresponding solution $z=(u, \gamma)$ to (6). Then, the solution blows up in finite time if and only if

$$
\liminf _{t \rightarrow T, x \in \mathbb{R}} u_{x}(t, x)=-\infty
$$

or

$$
\limsup _{t \rightarrow T}\left\{\left\|\gamma_{x}(t, \cdot)\right\|_{L^{\infty}}\right\}=+\infty .
$$

Proof. Multiplying the first equation in (5) by $m=u-u_{x x}$ and integrating by parts, we obtain

$$
\begin{aligned}
\frac{d}{d t} & \int_{\mathbb{R}} m^{2} d x \\
& =2 \int_{\mathbb{R}} m m_{t} d x \\
& =2 \int_{\mathbb{R}} m\left(-u m_{x}-2 m u_{x}-\rho \gamma_{x}\right) d x-2 \lambda \int_{\mathbb{R}} m^{2} d x \\
& =-3 \int_{\mathbb{R}} m^{2} u_{x} d x-2 \int_{\mathbb{R}} m \rho \gamma_{x} d x-2 \lambda \int_{\mathbb{R}} m^{2} d x .
\end{aligned}
$$

Repeating the same procedure to the second equation in (5), we get

$$
\frac{d}{d t} \int_{\mathbb{R}} \rho^{2} d x=-\int_{\mathbb{R}} \rho^{2} u_{x}-2 \lambda \int_{\mathbb{R}} \rho^{2} d x .
$$

A combination of (17) and (18) yields

$$
\begin{aligned}
\frac{d}{d t} \int_{\mathbb{R}}\left(m^{2}+\rho^{2}\right) d x= & -3 \int_{\mathbb{R}} m^{2} u_{x} d x-2 \int_{\mathbb{R}} m \rho \gamma_{x} d x \\
& -\int_{\mathbb{R}} \rho^{2} u_{x}-2 \lambda \int_{\mathbb{R}}\left(m^{2}+\rho^{2}\right) d x .
\end{aligned}
$$


Differentiating the first equation in (5) with respect to $x$, multiplying by $m_{x}=u_{x}-u_{x x x}$, and then integrating over $\mathbb{R}$, we obtain

$$
\begin{aligned}
\frac{d}{d t} \int_{\mathbb{R}} m_{x}^{2} d x= & -5 \int_{\mathbb{R}} m_{x}^{2} u_{x} d x+2 \int_{\mathbb{R}} m^{2} u_{x} d x \\
& -2 \int_{\mathbb{R}} m_{x} \rho_{x} \gamma_{x} d x-2 \int_{\mathbb{R}} m_{x} \rho \gamma_{x x} d x \\
& -2 \lambda \int_{\mathbb{R}} m_{x}^{2} d x .
\end{aligned}
$$

Similarly,

$$
\frac{d}{d t} \int_{\mathbb{R}} \rho_{x}^{2} d x=-3 \int_{\mathbb{R}} \rho_{x}^{2} u_{x} d x+\int_{\mathbb{R}} \rho^{2} u_{x x x} d x-2 \lambda \int_{\mathbb{R}} \rho_{x}^{2} d x
$$

A combination of (17)-(21) yields

$$
\begin{aligned}
& \frac{d}{d t} \int_{\mathbb{R}}\left(m^{2}+\rho^{2}+m_{x}^{2}+\rho_{x}^{2}\right) d x \\
& =-\int_{\mathbb{R}} m^{2} u_{x} d x-5 \int_{\mathbb{R}} m_{x}^{2} u_{x} d x \\
& -2 \int_{\mathbb{R}} m \rho \gamma_{x} d x-2 \int_{\mathbb{R}} m_{x} \rho_{x} \gamma_{x} d x \\
& -2 \lambda \int_{\mathbb{R}}\left(m^{2}+\rho^{2}\right) d x-2 \int_{\mathbb{R}} m_{x} \rho \gamma_{x x} d x \\
& -\int_{\mathbb{R}} \rho^{2} u_{x} d x-3 \int_{\mathbb{R}} \rho_{x}^{2} u_{x} d x \\
& +\int_{\mathbb{R}} \rho^{2} u_{x x x} d x-2 \lambda \int_{\mathbb{R}}\left(m_{x}^{2}+\rho_{x}^{2}\right) d x \\
& =-\int_{\mathbb{R}} m^{2} u_{x} d x-5 \int_{\mathbb{R}} m_{x}^{2} u_{x} d x \\
& -\int_{\mathbb{R}} \rho^{2} u_{x} d x-3 \int_{\mathbb{R}} \rho_{x}^{2} u_{x} d x \\
& -2 \lambda \int_{\mathbb{R}}\left(m^{2}+\rho^{2}\right) d x+\int_{\mathbb{R}} \rho^{2} u_{x x x} d x \\
& -2 \int_{\mathbb{R}} m \rho \gamma_{x} d x-2 \int_{\mathbb{R}} m_{x} \rho_{x} \gamma_{x} d x \\
& -2 \int_{\mathbb{R}} m_{x} \rho \gamma_{x x} d x-2 \lambda \int_{\mathbb{R}}\left(m_{x}^{2}+\rho_{x}^{2}\right) d x .
\end{aligned}
$$

Assume that there exist $M_{1}>0$ and $M_{2}>0$ such that $u x(t, x) \geq M_{1}$ and $\left\|\gamma_{x}(t, \cdot)\right\|_{L^{\infty}} \leq M_{2}$ for all $(t, x) \in[0, T) \times R$; then it follows from Lemma 4 that

$$
\|\rho(t, \cdot)\|_{L^{\infty}} \leq e^{M_{1} T}\left\|\rho_{0}(\cdot)\right\|_{L^{\infty}}
$$

Therefore,

$$
\begin{aligned}
& \frac{d}{d t} \int_{\mathbb{R}}\left(m^{2}+\rho^{2}+m_{x}^{2}+\rho_{x}^{2}\right) d x \\
& \leq\left(5 M_{1}+2 \lambda\right) \int_{\mathbb{R}}\left(m^{2}+\rho^{2}+m_{x}^{2}+\rho_{x}^{2}\right) d x \\
& \quad+\left(M_{2}+e^{M_{1} T}\left\|\rho_{0}(\cdot)\right\|_{L^{\infty}}\right) \\
& \quad \times \int_{\mathbb{R}}\left(m^{2}+\rho^{2}+m_{x}^{2}+\rho_{x}^{2}+u_{x x x}^{2}+\gamma_{x x}^{2}\right) d x \\
& \leq\left(5 M_{1}+2 \lambda\right) \int_{\mathbb{R}}\left(m^{2}+\rho^{2}+m_{x}^{2}+\rho_{x}^{2}\right) d x \\
& \quad+2\left(M_{2}+e^{M_{1} T}\left\|\rho_{0}(\cdot)\right\|_{L^{\infty}}\right) \\
& \quad \times \int_{\mathbb{R}}\left(m^{2}+\rho^{2}+m_{x}^{2}+\rho_{x}^{2}\right) d x \\
& \leq\left(5 M_{1}+2 \lambda\right)+2\left(M_{2}+e^{M_{1} T}\left\|\rho_{0}(\cdot)\right\|_{L^{\infty}}\right) \\
& \quad \times \int_{\mathbb{R}}\left(m^{2}+\rho^{2}+m_{x}^{2}+\rho_{x}^{2}\right) d x .
\end{aligned}
$$

The previous discussion shows that if there exist $M_{1}>0$ and $M_{2}>0$ such that $u_{x}(t, x) \geq M_{1}$ and $\left\|\gamma_{x}(t, \cdot)\right\| \leq M_{2}$ for all $(t, x) \in[0, T) \times \mathbb{R}$, then there exist two positive constants $K$ and $k$ such that the following estimate holds:

$$
\|u(t, \cdot)\|_{H^{s}}^{2}+\|v(t, \cdot)\|_{H^{s}}^{2} \leq K e^{k t}, \quad t \in[0, T) .
$$

This inequality, Sobolev's embedding theorem, and Theorem 2 guarantee that the solution does not blow up in finite time.

On the other hand, we see that, if

$$
\begin{gathered}
\liminf _{t \rightarrow T, x \in \mathbb{R}} u_{x}(t, x)=-\infty \text { or } \\
\limsup _{t \rightarrow T}\left\{\left\|\gamma_{x}(t, \cdot)\right\|_{L^{\infty}}\right\}=+\infty,
\end{gathered}
$$

then, by Sobolev's embedding theorem, the solution will blow up in finite time. This completes the proof of the theorem.

\section{Blow-Up Results and Blow-Up Rate Estimate}

In this section, we investigate the blow-up phenomena of strong solutions to (6). We now present the first blow-up result.

Lemma 6. Let $z_{0}=\left(u_{0}, \gamma_{0}\right) \in H^{s} \times H^{s}, s>5 / 2$, and let $T$ be the maximal existence time of the solution $z=(u, \gamma)$ to $(6)$ with the initial data $z_{0}$. Then, for all $t \in[0, T)$, one has

$$
\|u(t, \cdot)\|_{H^{1}}^{2}+\|\gamma(t, \cdot)\|_{H^{1}}^{2}=e^{-2 \lambda t}\left(\left\|u_{0}\right\|_{H^{1}}^{2}+\left\|\gamma_{0}\right\|_{H^{1}}^{2}\right) .
$$




\section{Moreover,}

$$
\begin{aligned}
& \|u(t, \cdot)\|_{L^{\infty}} \leq \frac{\sqrt{2}}{2}\|u(t, \cdot)\|_{H^{1}} \leq \frac{\sqrt{2}}{2}\left(\left\|u_{0}\right\|_{H^{1}}^{2}+\left\|\gamma_{0}\right\|_{H^{1}}^{2}\right)^{1 / 2}, \\
& \|\gamma(t, \cdot)\|_{L^{\infty}} \leq \frac{\sqrt{2}}{2}\|\gamma(t, \cdot)\|_{H^{1}} \leq \frac{\sqrt{2}}{2}\left(\left\|u_{0}\right\|_{H^{1}}^{2}+\left\|\gamma_{0}\right\|_{H^{1}}^{2}\right)^{1 / 2} .
\end{aligned}
$$

Proof. Denote

$$
\begin{gathered}
f(u, \gamma)=u^{2}+\frac{1}{2} u_{x}^{2}+\frac{1}{2} \gamma^{2}-\frac{1}{2} \gamma_{x}^{2}, \\
g=g(u, \gamma)=\left(u_{x} \gamma_{x}\right)_{x}+u_{x} \gamma .
\end{gathered}
$$

In view of the identity $-\partial_{x}^{2} p * f=f-p * f$, we can obtain, from (6),

$$
\begin{gathered}
u_{t x}=-u_{x}^{2}-u u_{x x}+f-p * f, \\
v_{t x}=-u_{x} \gamma_{x}-u \gamma_{x x}-\partial_{x} p * g .
\end{gathered}
$$

Therefore, an integration by parts yields

$$
\begin{aligned}
& \frac{1}{2} \frac{d}{d t}\left(\|u\|_{H^{1}}^{2}+\|\gamma\|_{H^{1}}^{2}\right) \\
& =\int_{\mathbb{R}}\left(u u_{t}+u_{x} u_{t x}+\gamma \gamma_{t}+\gamma_{x} \gamma_{t x}\right) d x \\
& =\int_{\mathbb{R}} u\left(-u u_{x}-\partial_{x}^{2} p * f-\lambda u\right) \\
& \quad+u_{x}\left(-u_{x}^{2}-u u_{x x}+f-p * f-\lambda u_{x}\right) \\
& \quad+\gamma\left(-u \gamma_{x}-\lambda \gamma\right) \\
& \quad+\gamma_{x}\left(-u \gamma_{x}-u \gamma_{x x}-\partial_{x} p * g-\lambda \gamma_{x}\right) d x \\
& =\int_{\mathbb{R}}\left[-\frac{1}{2} u_{x}^{3}+u_{x}\left(u^{2}+\frac{1}{2} u_{x}^{2}+\frac{1}{2} \gamma^{2}-\frac{1}{2} \gamma_{x}^{2}\right)\right. \\
& \quad-u \gamma \gamma_{x}-\gamma\left(u_{x x} \gamma_{x}+u_{x} \gamma\right)-u \gamma_{x} \gamma_{x}^{2} \\
& \left.\quad-u \gamma_{x} \gamma_{x x}-\lambda\left(u^{2}+u_{x}^{2}+\gamma^{2}+\gamma_{x}^{2}\right)\right] d x \\
& =-\lambda \int_{\mathbb{R}}\left(u^{2}+u_{x}^{2}+\gamma^{2}+\gamma_{x}^{2}\right) d x .
\end{aligned}
$$

Thus, the statement of the conservation law follows. The remaining part of this lemma can be easily deduced from the conservation law. The proof of the lemma is complete.

Lemma 7 (see [37]). Let $T>0$ and $v \in C^{1}\left([0, T) ; H^{2}\right)$. Then, for every $t \in[0, T)$, there exists at least one point $\xi \in R$ with

$$
m(t):=\inf _{x \in R}\left[v_{x}(t, x)\right]=v_{x}(t, \xi(t)) .
$$

The function $m(t)$ is absolutely continuous on $(0, T)$ with

$$
\frac{d m}{d t}=v_{t x}(t, \xi(t)), \quad \text { a.e., on }(0, T) \text {. }
$$

Theorem 8. Let $z_{0}=\left(u_{0}, \gamma_{0}\right) \in H^{s} \times H^{s}, s>5 / 2$, and let $T$ be the maximal existence time of the solution $z=(u, \gamma)$ to the (6) with the initial data $z_{0}$. If there exists some $x_{0} \in \mathbb{R}$ such that

$$
u_{0}^{\prime}\left(x_{0}\right)<-\lambda-\left[\lambda^{2}+\left(\left\|u_{0}\right\|_{H^{1}}^{2}+\left\|\gamma_{0}\right\|_{H^{1}}^{2}\right)\right]^{1 / 2}
$$

then the existence time $T$ is finite and the slope of $u$ tends to negative infinity as $t$ goes to $T$ while $u$ remains uniformly bounded on $[0, T]$.

Proof. As mentioned earlier, here we only need to show that the previous theorem holds for $s=3$. Differentiating the first equation of (6) with respect to $x$, in view of $\partial_{x}^{2} p * f=p * f-f$, we have

$$
\begin{aligned}
u_{t x}+u u_{x x}= & -\frac{1}{2} u_{x}^{2}+u^{2}+\frac{1}{2} \gamma^{2}-\frac{1}{2} \gamma_{x}^{2} \\
& -p *\left(u^{2}+\frac{1}{2} u_{x}^{2}+\frac{1}{2} \gamma^{2}-\frac{1}{2} \gamma_{x}^{2}\right) \\
& -\lambda u_{x} .
\end{aligned}
$$

Note that

$$
\begin{aligned}
& \frac{d u_{x}(t, q(t, x))}{d t} \\
& =u_{x t}(t, q(t, x))+u_{x x}(t, q(t, x)) q_{t}(t, x) \\
& =u_{x t}(t, q(t, x))+u(t, q(t x)) u_{x x}(t, q(t, x)) .
\end{aligned}
$$

We know that $p *\left(u^{2}+(1 / 2) u_{x}^{2}\right) \geq(1 / 2) u^{2}$ and

$$
\left\|p * \gamma_{x}^{2}\right\|_{L^{\infty}} \leq\|p\|_{L^{\infty}}\left\|\gamma_{x}^{2}\right\|_{L^{1}}=\frac{1}{2}\left\|\gamma_{x}^{2}\right\|_{L^{1}}
$$

By (35) and (36) and the previous estimates, we deduce that

$$
\begin{aligned}
& \frac{d u_{x}(t, q(t, x))}{d t} \\
& \leq-\frac{1}{2} u_{x}^{2}(t, q(t, x))+\frac{1}{2} u^{2}(t, q(t, x)) \\
& +\frac{1}{4} \gamma^{2}(t, q(t, x)) \\
& +\frac{3}{4} p *\left(\gamma_{x}^{2}\right)(t, q(t, x))-\lambda u_{x} \\
& \leq-\frac{1}{2} u_{x}^{2}(t, q(t, x))+\frac{1}{2} u^{2}(t, q(t, x)) \\
& +\frac{1}{4} \gamma^{2}(t, q(t, x))+\frac{3}{8}\left\|\gamma_{x}\right\|_{L^{1}}-\lambda u_{x}
\end{aligned}
$$




$$
\begin{aligned}
\leq & -\frac{1}{2} u_{x}^{2}(t, q(t, x))+\frac{1}{4}\|u\|_{H^{1}}^{2} \\
& +\frac{1}{8}\|\gamma\|_{H^{1}}^{2}+\frac{3}{8}\left\|\gamma_{x}^{2}\right\|_{L^{1}}-\lambda u_{x} \\
\leq & -\frac{1}{2} u_{x}^{2}(t, q(t, x)) \\
& +\frac{1}{2}\left(\|u\|_{H^{1}}^{2}+\|\gamma\|_{H^{1}}^{2}\right)-\lambda u_{x} \\
= & -\frac{1}{2} u_{x}^{2}(t, q(t, x))-\lambda u_{x} \\
& +\frac{1}{2}\left(\left\|u_{0}\right\|_{H^{1}}^{2}+\left\|\gamma_{0}\right\|_{H^{1}}^{2}\right)
\end{aligned}
$$

in view of Lemma 6 . Take

$$
K=\frac{\sqrt{2}}{2}\left(\left\|u_{0}\right\|_{H^{1}}^{2}+\left\|\gamma_{0}\right\|_{H^{1}}^{2}\right)^{1 / 2}
$$

and define $g(t)=u_{x}\left(t, q\left(t, x_{0}\right)\right)$. It then follows from (38) that on $[0, T)$,

$$
\begin{aligned}
g^{\prime}(t) \leq & -\frac{1}{2} g^{2}(t)-\lambda g+K^{2} \\
= & -\frac{1}{2}\left(g(t)+\lambda+\sqrt{\lambda^{2}+2 K^{2}}\right) \\
& \times\left(g(t)+\lambda-\sqrt{\lambda^{2}+2 K^{2}}\right) .
\end{aligned}
$$

Note that if $g(0) \leq-\lambda-\sqrt{\lambda^{2}+2 K^{2}}$, then $g(t) \leq-\lambda-$ $\sqrt{\lambda^{2}+2 K^{2}}$, for all $t \in[0, T)$. Therefore, we can solve the previous inequality to obtain

$$
\begin{gathered}
\frac{g(0)+\lambda+\sqrt{\lambda^{2}+2 K^{2}} e^{\sqrt{\lambda^{2}+2 K^{2} t}}-1}{g(0)+\lambda-\sqrt{\lambda^{2}+2 K^{2}}} \\
\leq \frac{2 \sqrt{\lambda^{2}+2 K^{2}}}{g(t)+\lambda-\sqrt{\lambda^{2}+2 K^{2}}} .
\end{gathered}
$$

Due to $0<\left(g(0)+\lambda+\sqrt{\lambda^{2}+2 K^{2}}\right) /\left(g(0)+\lambda-\sqrt{\lambda^{2}+2 K^{2}}\right)<1$, then there exists $T$, and $0<T<\left(1 / \sqrt{\lambda^{2}+2 K^{2}}\right) \ln ((g(0)+\lambda+$ $\left.\left.\sqrt{\lambda^{2}+2 K^{2}}\right) /\left(g(0)+\lambda-\sqrt{\lambda^{2}+2 K^{2}}\right)\right)$, such that $\lim _{t \rightarrow T} g(t)=$ $-\infty$. Applying Theorem 5 , the solution $z$ does not exist globally in time.

Next, we give a blow-up result if $u_{0}$ and $\gamma_{0}$ are odd.

Theorem 9. Let $z_{0}=\left(u_{0}, \gamma_{0}\right) \in H^{s} \times H^{s}, s>5 / 2$, and let $T$ be the maximal existence time of the solution $z=(u, \gamma)$ to $(6)$ with the initial data $z_{0}$. If $u_{0}$ and $\gamma_{0}$ are odd, and furthermore

$$
u_{0}^{\prime}\left(x_{0}\right)<-\lambda-\left[\lambda^{2}+\frac{1}{2}\left(\left\|u_{0}\right\|_{H^{1}}^{2}+\left\|\gamma_{0}\right\|_{H^{1}}^{2}\right)\right]^{1 / 2},
$$

then $T$ is finite and $u_{x}(t, 0) \rightarrow-\infty$ as $t$ goes to $T$.
Proof. As mentioned earlier, here we only need to show that the previous theorem holds for $s=3$. Note that (6) is the invariant under the transformation $(u, x) \rightarrow(-u,-x)$ and $(\gamma, x) \rightarrow(-\gamma,-x)$. Thus, we deduce that if $u_{0}(x)$ and $\gamma_{0}(x)$ are odd, then $u(t, x)$ and $\gamma(t, x)$ are odd for any $t \in[0, T)$. By continuity with respect to $x$ of $z$ and $z_{x x}$, we have

$$
u(t, 0)=u_{x x}(t, 0)=\gamma(t, 0)=\gamma_{x x}(t, 0)=0, \quad \forall t \in[0, T) .
$$

Hence, in view of (35) and Lemma 6, we obtain

$$
\begin{aligned}
u_{t x}(t, 0)= & -\frac{1}{2} u_{x}^{2}(t, 0)-\frac{1}{2} \gamma_{x}^{2} \\
& -p *\left(u^{2}+\frac{1}{2} u_{x}+\frac{1}{2} \gamma^{2}-\frac{1}{2} \gamma_{x}^{2}\right)(t, 0)-\lambda u_{x} \\
\leq & -\frac{1}{2} u_{x}^{2}(t, 0)+\frac{1}{2} p * \gamma_{x}^{2}(t, 0)-\lambda u_{x} \\
\leq & -\frac{1}{2} u_{x}^{2}(t, 0)-\lambda u_{x}+\frac{1}{4}\left(\left\|u_{0}\right\|_{H^{1}}^{2}+\left\|\gamma_{0}\right\|_{H^{1}}^{2}\right)
\end{aligned}
$$

Take

$$
K=\frac{1}{2}\left(\left\|u_{0}\right\|_{H^{1}}^{2}+\left\|\gamma_{0}\right\|_{H^{1}}^{2}\right)^{1 / 2}
$$

and define $g(t)=u_{x}\left(t, q\left(t, x_{0}\right)\right)$. It then follows from (38) that on $[0, T)$,

$$
\begin{aligned}
g^{\prime}(t) \leq & -\frac{1}{2} g^{2}(t)-\lambda g+K^{2} \\
= & -\frac{1}{2}\left(g(t)+\lambda+\sqrt{\lambda^{2}+2 K^{2}}\right) \\
& \times\left(g(t)+\lambda-\sqrt{\lambda^{2}+2 K^{2}}\right) .
\end{aligned}
$$

Note that if $g(0) \leq-\lambda-\sqrt{\lambda^{2}+2 K^{2}}$, then $g(t) \leq-\lambda-$ $\sqrt{\lambda^{2}+2 K^{2}}$, for all $t \in[0, T)$. Therefore, we can solve the previous inequality to obtain

$$
\begin{gathered}
\frac{g(0)+\lambda+\sqrt{\lambda^{2}+2 K^{2}} e^{\sqrt{\lambda^{2}+2 K^{2} t}}-1}{g(0)+\lambda-\sqrt{\lambda^{2}+2 K^{2}}} \\
\leq \frac{2 \sqrt{\lambda^{2}+2 K^{2}}}{g(t)+\lambda-\sqrt{\lambda^{2}+2 K^{2}}} .
\end{gathered}
$$

Due to $0<\left(g(0)+\lambda+\sqrt{\lambda^{2}+2 K^{2}}\right) /\left(g(0)+\lambda-\sqrt{\lambda^{2}+2 K^{2}}\right)<1$, then there exists $T$, and $0<T<\left(1 / \sqrt{\lambda^{2}+2 K^{2}}\right) \ln ((g(0)+\lambda+$ $\left.\left.\sqrt{\lambda^{2}+2 K^{2}}\right) /\left(g(0)+\lambda-\sqrt{\lambda^{2}+2 K^{2}}\right)\right)$, such that $\lim _{t \rightarrow T} g(t)=$ $-\infty$. Applying Theorem 5, the solution $z$ does not exist globally in time.

Next, we give more insight into the blow-up rate for the wave-breaking solutions to (6).

Theorem 10. Let $z_{0}=\left(u_{0}, \gamma_{0}\right) \in H^{s} \times H^{s}, s \geq 5 / 2, z=(u, \gamma)$ be the corresponding solution to (6) with initial data $z_{0}$ and 
satisfy $\left\|\gamma_{x}(t, x)\right\|_{L^{\infty}} \leq M$, for all $(t, x) \in[0, T) \times R$, and $T$ be the maximal existence time of the solution. Then let one has

$$
\lim _{t \rightarrow T}\left(\inf _{x \in \mathbb{R}}\left(u_{x}(t, x)(T-t)\right)\right)=-2 .
$$

Proof. By Lemma 6, we get the uniform bound of $u$. Set $m(t)=\inf _{x \in \mathbb{R}} u_{x}(t, x)$. By the proof of Theorem 8 (or Theorem 9), we find a constant $K>0$ such that

$$
\left|g^{\prime}(t)+\frac{1}{2} g(t)+\lambda g(t) \leq K\right|,
$$

where $K$ depends only on $\left\|u_{0}\right\|_{H^{1}}$ and $\left\|\gamma_{0}\right\|_{H^{1}}$. It follows that

$$
\begin{aligned}
-K-\frac{1}{2} \lambda^{2} & \leq g^{\prime}(t)+\frac{1}{2}(g(t)+\lambda)^{2} \\
& \leq K+\frac{1}{2} \lambda^{2}, \quad \text { a.e., on }(0, T) .
\end{aligned}
$$

Choose $\epsilon \in(0,1 / 2)$. Since $\liminf _{r \rightarrow T}(y(t)+\lambda)=-\infty$ by Theorem 5 , there is some $t_{0} \in(0, T)$ with $g\left(t_{0}\right)+\lambda<0$ and $\left(g\left(t_{0}\right)+\lambda\right)^{2}>\left(K+(1 / 2) \lambda^{2}\right) / \epsilon$. Let us first prove that

$$
(g(t)+\lambda)^{2}>\frac{1}{\epsilon}\left(K+\frac{1}{2} \lambda^{2}\right), \quad t \in\left[t_{0}, T\right) .
$$

Since $g$ is locally Lipschitz, there is some $\delta>0$ such that

$$
(g(t)+\lambda)^{2}>\frac{1}{\epsilon}\left(K+\frac{1}{2} \lambda^{2}\right), \quad t \in\left(t_{0}, t_{0}+\delta\right) .
$$

Note that $g$ is locally Lipschitz and therefore absolutely continuous. Integrating the previous relation on $\left(t_{0}, t_{0}+\delta\right)$ yields that

$$
g\left(t_{0}+\delta\right)+\lambda \leq g\left(t_{0}\right)+\lambda<0 .
$$

It follows from the previous inequality that

$$
\left(g\left(t_{0}+\delta\right)+\lambda\right)^{2} \geq\left(g\left(t_{0}\right)+\lambda\right)^{2}>\frac{1}{\epsilon}\left(K+\frac{1}{2} \lambda^{2}\right) .
$$

By (50)-(51), we infer that

$$
\frac{1}{2}-\epsilon \leq-\frac{g^{\prime}(t)}{(m+\lambda)^{2}} \leq \frac{1}{2}+\epsilon, \quad \text { a.e., on }(0, T) .
$$

For $t \in\left(t_{0}, T\right)$, integrating $(55)$ on $(t, T)$ to get

$$
\begin{aligned}
\left(\frac{1}{2}-\epsilon\right)(T-t) & \leq-\frac{1}{g(t)+\lambda} \\
& \leq\left(\frac{1}{2}+\epsilon\right)(T-t), \quad t \in\left(t_{0}, T\right) .
\end{aligned}
$$

Since $g(t)+\lambda<0$ on $\left[t_{0}, T\right)$, it follows that

$$
\begin{aligned}
\frac{1}{(1 / 2)+\epsilon} & \leq-(g(t)+\lambda)(T-t) \\
& \leq \frac{1}{(1 / 2)+\epsilon}, \quad t \in\left(t_{0}, T\right) .
\end{aligned}
$$

By the arbitrariness of $\epsilon \in(0,1 / 2)$, the statement of the theorem follows.

\section{Asymptotic Profile}

In this section, we focus on the persistence property of the solution to (6) in $L^{\infty}$-space. Precisely, we give an asymptotic description on how the solutions behave under the initial values possess algebraic decay at infinity. Recently, the asymptotic behavior for the celebrated Camassa-Holm equation was investigated in [38]. We notice that in [39], the authors showed that the solution of the Camassa-Holm equation and its first-order spatial derivative retain exponential decay at infinity as their initial values behave. After all, the exponential decay of initial value is a faster way; this motivates us to establish the decay rate of solution if its initial value decays algebraically. We show that the strong solution of (6) corresponding to initial data with a slower algebraically decaying way will keep this behavior in the $x$-variable at infinity in its lifespan. In order to achieve our result, we first recall the following lemma.

Lemma 11 (see [40]). For a function $\Phi_{N}(X)$ defined next, there exists a constant $C_{\theta}$ which only depends on $\theta \in(0,1]$, such that for any positive integer $N \geq 2$

$$
\Phi_{N}(x) \int_{R} e^{-|x-y|} \frac{1}{\Phi_{N}(y)} d y \leq C_{\theta},
$$

where

$$
\Phi_{N}(x)= \begin{cases}1, & x \leq 1 \\ x^{\theta}, & x \in(1, N) \\ N^{\theta}, & X \geq N .\end{cases}
$$

Theorem 12. Assume that $X_{0}(x)=\left(u_{0}(x), \gamma_{0}(x)\right)^{T} \in H^{s} \times H^{s}$ with $s>5 / 2$ satisfies that for some $\theta \in(0,1]$

$$
\left|X_{0}(x)\right|, \quad\left|X_{0 x}(x)\right| \sim O\left(x^{-\theta}\right) \quad \text { as } x \uparrow \infty \text {. }
$$

Then, the corresponding strong solution $X(x)=(u(x)$, $\gamma(x))^{T} \in C\left([0, T) ; H^{s} \times H^{s}\right)$ to $(6)$ satisfies that

$$
|X(x)|, \quad\left|X_{x}(x)\right| \sim O\left(x^{-\theta}\right) \quad \text { as } x \uparrow \infty,
$$

uniformly in the time interval $[0, T)$.

Notation. One has

$$
|f(x)| \sim O(|g(x)|) \quad \text { as } x \uparrow \infty \text { if } \lim _{x \rightarrow \infty} \frac{|f(x)|}{|g(x)|}=L,
$$

where $L$ is a nonnegative constant. In order to shorten the presentation in the sequel, we introduce

$$
\begin{gathered}
F(u, \gamma)=u^{2}+\frac{1}{2} u_{x}^{2}+\frac{1}{2} \gamma^{2}-\frac{1}{2} \gamma_{x}^{2}, \\
H(u, \gamma)=\left(u_{x} \gamma_{x}\right)_{x}+u_{x} \gamma .
\end{gathered}
$$

Proof. The first step is devoted to giving estimates on $\|u(x, t)\|_{\infty}$ and $\|\gamma(x, t)\|_{\infty}$, where $\|\cdot\|_{p}$ is the standard $L^{p}(\mathbb{R})$ norm. 
Multiplying the first equation of (6) by $u^{2 n-1}$ with $n \in$ $Z^{+}$and integrating both sides with respect to $x$ variable, we obtain

$$
\begin{aligned}
& \int_{\mathbb{R}} u^{2 n-1} u_{t} d x+\int_{\mathbb{R}} u^{2 n-1} u u_{x} d x \\
& \quad+\int_{\mathbb{R}} u^{2 n-1} \partial_{x}(G * F) d x+\lambda \int_{\mathbb{R}} u^{2 n} d x=0 .
\end{aligned}
$$

The first term in (64) is

$$
\int_{\mathbb{R}} u^{2 n-1} u_{t} d x=\frac{1}{2 n} \frac{d}{d t}\|u\|_{2 n}^{2 n}=\|u\|_{2 n}^{2 n-1} \frac{d}{d t}\|u\|_{2 n} ;
$$

for the second term of (64), we have

$$
\int_{\mathbb{R}} u^{2 n-1} u u_{x} d x \leq\left\|u_{x}\right\|_{\infty}\|u\|_{2 n}^{2 n} .
$$

It follows from the Höder inequality that

$$
\int_{\mathbb{R}} u^{2 n-1} \partial_{x}(G * F(u)) d x \leq\|u\|_{2 n}^{2 n-1}\left\|\partial_{x}(G * F)\right\|_{2 m} .
$$

Therefore,

$$
\frac{d}{d t}\|u\|_{2 n} \leq\left(\left\|u_{x}\right\|_{\infty}+\lambda\right)\|u\|_{2 n}+\left\|\partial_{x}(G * F)\right\|_{2 n} .
$$

Similarly, for the estimate of $\|\gamma(x)\|_{\infty}$, we have by multiplying $\gamma^{2 n-1}$ and integration

$$
\begin{aligned}
& \int_{\mathbb{R}} \gamma^{2 n-1} \gamma_{t} d x+\int_{\mathbb{R}} \gamma^{2 n-1} u \gamma_{x} d x \\
& \quad+\int_{\mathbb{R}} \gamma^{2 n-1}(G * H) d x+\lambda \int_{\mathbb{R}} \gamma^{2 n-1} d x=0, \\
& \frac{d}{d t}\|\gamma\|_{2 n} \leq\left(\left\|\gamma_{x}\right\|_{\infty}+\lambda\right)\|u\|_{2 n}+\|G * H\|_{2 n} .
\end{aligned}
$$

By the Sobolev embedding theorem, there exists a constant $M>0$ such that

$$
\begin{aligned}
\frac{d}{d t}\left(\|\gamma\|_{2 n}+\|u\|_{2 n}\right) \leq & M\left(\|\gamma\|_{2 n}+\|u\|_{2 n}\right) \\
& +\left\|\partial_{x}(G * H)\right\|_{2 n}+\|G * H\|_{2 n} .
\end{aligned}
$$

In view of Gronwall's inequality, we have the estimate

$$
\begin{aligned}
\|\gamma\|_{2 n}+\|u\|_{2 n} \\
\leq e^{M t}\left(\left\|\gamma_{0}\right\|_{2 n}+\left\|u_{0}\right\|_{2 n}\right. \\
\left.\quad+\int_{0}^{t}\left(\left\|\partial_{x}(G * H)\right\|_{2 n}+\|G * H\|_{2 n}\right) d \tau\right) .
\end{aligned}
$$

We can take limits as $N$ goes to infinity to obtain

$$
\begin{aligned}
& \|\gamma\|_{\infty}+\|u\|_{\infty} \\
& \leq e^{M t}\left(\left\|\gamma_{0}\right\|_{\infty}+\left\|u_{0}\right\|_{\infty}\right. \\
& \left.\quad+\int_{0}^{t}\left(\left\|\partial_{x}(G * H)\right\|_{\infty}+\|G * H\|_{\infty}\right) d \tau\right) .
\end{aligned}
$$

The second step is to establish estimates for $\left\|u_{x}\right\|_{\infty}$ and $\left\|\gamma_{x}\right\|_{\infty}$ by using the same method as previously mentioned. Differentiating the first equation of (6) with respect to $x$ produces the following equation:

$$
u_{x t}+u_{x}^{2}+u u_{x x}+\partial_{x}^{2}(G * F)+\lambda u_{x}=0 .
$$

Multiplying (73) by $u_{x}^{2 n-1}$, and then integrating by parts, one obtains

$$
\begin{gathered}
\int_{\mathbb{R}} u_{x}^{2 n-1} u_{x t} d x+\int_{\mathbb{R}} u_{x}^{2 n+1} d x-\frac{1}{2 n} \int_{\mathbb{R}} u_{x}^{2 n} u_{x} \\
+\int_{\mathbb{R}} u_{x}^{2 n-1} \partial_{x}^{2}(G * F) d x+\lambda \int_{\mathbb{R}} u_{x}^{2 n}=0 .
\end{gathered}
$$

Similarly, one can obtain

$$
\left\|u_{x}\right\|_{\infty} \leq e^{M t}\left(\left\|u_{0 x}\right\|_{\infty}+\int_{0}^{t}\left\|\partial_{x}^{2}(G * F)\right\|_{\infty} d \tau\right) .
$$

For the second equation of (6), we may get

$$
\begin{aligned}
\int_{\mathbb{R}} & \gamma_{x}^{2 n-1} \gamma_{x t} d x+\int_{\mathbb{R}} u_{x} \gamma_{x}^{2 n} d x \\
& +\int_{\mathbb{R}} u \gamma_{x x} \gamma_{x}^{2 n-1} d x+\int_{\mathbb{R}} \gamma_{x}^{2 n-1} \partial_{x}(G * H) d x \\
& +\lambda \int_{\mathbb{R}} \gamma_{x}^{2 n}=0, \\
\frac{d}{d t}\left\|\gamma_{x}\right\|_{2 n} \leq & \left(\left\|u_{x}\right\|_{\infty}+\lambda\right)\left\|\gamma_{x}\right\|_{2 n} \\
& +\left\|\gamma_{x x}\right\|_{\infty}\|u\|_{2 n}+\left\|\partial_{x}(G * H)\right\|_{2 n},
\end{aligned}
$$

and by Gronwall's inequality

$$
\left\|\gamma_{x}\right\|_{\infty} \leq e^{M t}\left(\left\|\gamma_{0 x}\right\|_{\infty}+\int_{0}^{t}\left(\|u\|_{\infty}+\left\|\partial_{x}(G * H)\right\|_{\infty}\right) d \tau\right) .
$$

In order to arrive at our result, we introduce a weighted continuous function which is independent on $t$ as follows:

$$
\Phi_{N}(x)= \begin{cases}1, & x \leq 1, \\ x^{\theta}, & x \in(1, N), \\ N^{\theta}, & X \geq N,\end{cases}
$$

where $\theta \in(0,1], N \in Z^{+}, N>2$. It is trivial that

$$
0 \leq \Phi_{N}^{\prime}(x) \leq \Phi_{N}(x), \quad \text { a.e., } x \in R,
$$

where the derivative is with respect to the variable $x$. From the first equation of (6) and (73), we have

$$
\begin{gathered}
\Phi_{N} u_{t}+\Phi_{N} u u_{x}+\Phi_{N} \partial_{x}(G * F)+\lambda \Phi_{N} u=0 \\
\Phi_{N} u_{x t}+\Phi_{N} u_{x}^{2}+\Phi_{N} u u_{x x}+\Phi_{N} \partial_{x}^{2}(G * F)+\lambda \Phi_{N} u_{x}=0
\end{gathered}
$$


Next, in order to obtain the estimates on $\left\|u \Phi_{N}\right\|_{\infty}$ and $\left\|u_{x} \Phi_{N}\right\|_{\infty}$, we apply a similar technique that was used before for $\|u(x, t)\|_{\infty}$ and $\left\|u_{x}(x, t)\right\|_{\infty}$ step by step to (80) and (81). For (81), we need to eliminate the term with the second-order derivative in order to attain the estimate for $u_{x} \Phi_{N}$. Using integration by parts, we obtain

$$
\begin{aligned}
& \left|\int_{\mathbb{R}}\left(u_{x} \Phi_{N}\right)^{2 n-1} \Phi_{N} u u_{x x} d x\right| \\
& \quad=\left|\int_{R}\left(u_{x} \Phi_{N}\right)^{2 n-1} u\left(\left(\Phi_{N} u_{x}\right)_{x}-\Phi_{N}^{\prime} u_{x}\right) d x\right| \\
& \quad=\left|\int_{\mathbb{R}} u\left(\frac{\left(u_{x} \Phi_{N}\right)^{2 n}}{2 n}\right)_{x} d x-\int_{\mathbb{R}} u\left(u_{x} \Phi_{N}\right)^{2 n-1} \Phi_{N}^{\prime} u_{x} d x\right| \\
& \quad \leq 2\left(\left\|u_{x}\right\|_{\infty}+\|u\|_{\infty}\right)\left\|u_{x} \Phi_{N}\right\|_{2 n}^{2 n},
\end{aligned}
$$

where (79) is used directly. Therefore, with these preparations, it holds that

$$
\begin{aligned}
& \left\|u \Phi_{N}\right\|_{\infty}+\left\|u_{x} \Phi_{N}\right\|_{\infty} \\
& \leq e^{2 M t}\left(\left\|u_{0} \Phi_{N}\right\|_{\infty}+\left\|u_{0 x} \Phi_{N}\right\|_{\infty}\right) \\
& \quad+e^{2 M t} \int_{0}^{t}\left(\left\|\Phi_{N} \partial_{x}(G * H)\right\|_{\infty}+\left\|\Phi_{N} \partial_{x}^{2}(G * F)\right\|_{\infty}\right) d \tau .
\end{aligned}
$$

For the second equation of (6), we have

$$
\begin{gathered}
\Phi_{N} \gamma_{t}+\Phi_{N} u \gamma_{x}+\Phi_{N} G * H+\lambda \gamma=0, \\
\Phi_{N} \gamma_{x t}+\Phi_{N} u_{x} \gamma_{x}+\Phi_{N} u \gamma_{x x} \\
+\Phi_{N} \partial_{x} G * H+\lambda \gamma_{x}=0 .
\end{gathered}
$$

Therefore,

$$
\begin{aligned}
& \frac{d}{d t}\left\|\gamma \Phi_{N}\right\|_{2 n} \leq\|u\|_{\infty}\left\|\Phi_{N} \gamma\right\|_{2 n}+\frac{1}{2 n}\left\|u_{x}\right\|_{\infty}\left\|\gamma \Phi_{N}\right\|_{2 n} \\
& +\left\|\Phi_{N} G * H\right\|_{2 n} \\
& \frac{d}{d t}\left\|\gamma_{x} \Phi_{N}\right\|_{2 n} \leq 3\left(\|u\|_{\infty}+\left\|u_{x}\right\|_{\infty}\right)\left\|\gamma_{x} \Phi_{N}\right\|_{2 n} \\
& +\left\|\Phi_{N} \partial_{x}(G * H)\right\|_{2 n} .
\end{aligned}
$$

Then by (85), we obtain

$$
\begin{aligned}
& \left\|\gamma \Phi_{N}\right\|_{\infty}+\left\|\gamma_{x} \Phi_{N}\right\|_{\infty} \\
& \leq e^{3 M t}\left(\left\|\gamma_{0} \Phi_{N}\right\|_{\infty}+\left\|\gamma_{0 x} \Phi_{N}\right\|_{\infty}\right) \\
& \quad+e^{3 M t} \int_{0}^{t}\left(\left\|\Phi_{N} \partial_{x}(G * H)\right\|_{\infty}+\left\|\Phi_{N} \partial_{x}^{2}(G * F)\right\|_{\infty}\right) d \tau .
\end{aligned}
$$

On the other hand, for a suitable function $f$, one obtains, due to Lemma 11,

$$
\begin{aligned}
& \left|\Phi_{N} \partial_{x}\left(G * f^{2}\right)\right| \\
& \quad \leq \Phi_{N}(x) \int_{R} e^{-|x-y|} \frac{1}{\Phi_{N}(y)} \Phi_{N}(y) f(y) f(y) d y \\
& \quad \leq\left\|f \Phi_{N}\right\|_{\infty}\|f\|_{\infty} \Phi_{N}(x) \int_{R} e^{-|x-y|} \frac{1}{\Phi_{N}(y)} d y \\
& \quad \leq C_{\theta}\left\|f \Phi_{N}\right\|_{\infty}\|f\|_{\infty} .
\end{aligned}
$$

Similarly,

$$
\left|\Phi_{N} \partial_{x}^{2}\left(G * f^{2}\right)\right| \leq C_{\theta}\left\|f \Phi_{N}\right\|_{\infty}\|f\|_{\infty} .
$$

Note that there are two additional quantities in (86) to be dealt with. Let us estimate $\left\|\Phi_{N}(G * H)\right\|_{\infty}$ first. One has

$$
\begin{aligned}
& \left|\Phi_{N}(G * H)\right| \\
& \quad=\frac{1}{2}\left|\Phi_{N}(x) \int_{R} e^{-|x-y|}\left(\left(u_{x} \gamma_{x}\right)_{x}+u_{x} \gamma\right) d y\right| \\
& \quad \leq C_{\theta}\left\|u_{x} \Phi_{N}\right\|_{\infty}\left(\|\gamma\|_{\infty}+\left\|\gamma_{x}\right\|_{\infty}\right) .
\end{aligned}
$$

It is similar to the remaining $\left\|\Phi_{N} \partial_{x}(G * H)\right\|_{\infty}$. Then, combining (87)-(89) with (83) and (86), it follows that there exists a constant $\widetilde{C}=\widetilde{C}(M, T)>0$ such that

$$
\begin{aligned}
\Gamma(t) & \leq \widetilde{C} \Gamma(0)+\widetilde{C} \int_{0}^{t} \zeta(\tau) \Gamma(\tau) d \tau \\
& \leq \widetilde{C}\left(\Gamma(0)+\int_{0}^{t} \Gamma(\tau) d \tau\right),
\end{aligned}
$$

where

$$
\begin{gathered}
\Gamma(t)=\left\|u \Phi_{N}\right\|_{\infty}+\left\|u_{x} \Phi_{N}\right\|_{\infty}+\gamma \Phi_{N}\left\|_{\infty}+\gamma_{x} \Phi_{N}\right\|_{\infty}, \\
\zeta(t)=\|u\|_{\infty}+\left\|u_{x}\right\|_{\infty}+\|\gamma\|_{\infty}+\left\|\gamma_{x}\right\|_{\infty}
\end{gathered}
$$

are introduced just for simplicity. Next for any $N \in Z^{+}, t \in$ $[0, T]$, and $x>0$, we have, by Gronwall's inequality,

$$
\begin{aligned}
\Gamma(t) \leq & \widetilde{C}_{0} \Gamma(0) \\
\leq & \widetilde{C}_{0}\left(\left\|u_{0}(x) x^{\theta}\right\|_{\infty}+\left\|u_{0 x}(x) x^{\theta}\right\|_{\infty}\right. \\
& \left.\quad+\left\|\gamma_{0}(x) x^{\theta}\right\|_{\infty}+\left\|\gamma_{0 x}(x) x^{\theta}\right\|_{\infty}\right) .
\end{aligned}
$$

Finally, passing limit as $N$ goes to infinity in the previous inequality, we obtain

$$
\begin{aligned}
& \left\|u(x, t) x^{\theta}\right\|_{\infty}+\left\|u_{x}(x, t) x^{\theta}\right\|_{\infty} \\
& +\left\|\gamma(x, t) x^{\theta}\right\|_{\infty}+\left\|\gamma_{x}(x, t) x^{\theta}\right\|_{\infty} \\
& \leq\left\|u_{0}(x) x^{\theta}\right\|_{\infty}+\left\|u_{0 x}(x) x^{\theta}\right\|_{\infty} \\
& +\left\|\gamma_{0}(x) x^{\theta}\right\|_{\infty}+\left\|\gamma_{0}(x) x^{\theta}\right\|_{\infty} .
\end{aligned}
$$

We complete the proof. 


\section{Acknowledgments}

The authors would like to say thanks to Professor Zhaoyang Yin and Professor Yong Zhou for sending several of their papers to the authors. This work was partially supported by NSF of China (11371384), NSF of Chong qing (cstc2013jcyjA0940), and NSF of Fuling (FLKJ, 2013ABA2036).

\section{References}

[1] R. Camassa and D. D. Holm, "An integrable shallow water equation with peaked solitons," Physical Review Letters, vol. 71, no. 11, pp. 1661-1664, 1993.

[2] M. Chen, S.-Q. Liu, and Y. Zhang, "A two-component generalization of the Camassa-Holm equation and its solutions," Letters in Mathematical Physics, vol. 75, no. 1, pp. 1-15, 2006.

[3] Z. Popowicz, "A 2-component or $N=2$ supersymmetric Camassa-Holm equation," Physics Letters A, vol. 354, no. 1-2, pp. 110-114, 2006.

[4] H. Holden and X. Raynaud, "Global conservative solutions of the Camassa-Holm equation-a Lagrangian point of view," Communications in Partial Differential Equations, vol. 32, no. 10-12, pp. 1511-1549, 2007.

[5] H. Holden and X. Raynaud, "Dissipative solutions for the Camassa-Holm equation," Discrete and Continuous Dynamical Systems A, vol. 24, no. 4, pp. 1047-1112, 2009.

[6] Y. Zhou, "Stability of solitary waves for a rod equation," Chaos, Solitons and Fractals, vol. 21, no. 4, pp. 977-981, 2004.

[7] Z. Jiang, L. Ni, and Y. Zhou, "Wave breaking of the CamassaHolm equation," Journal of Nonlinear Science, vol. 22, no. 2, pp. 235-245, 2012.

[8] Y. Zhou and H. Chen, "Wave breaking and propagation speed for the Camassa-Holm equation with $\kappa \neq 0$," Nonlinear Analysis. Real World Applications. An International Multidisciplinary Journal, vol. 12, no. 3, pp. 1875-1882, 2011.

[9] A. Constantin and R. I. Ivanov, "On an integrable twocomponent Camassa-Holm shallow water system," Physics Letters A, vol. 372, no. 48, pp. 7129-7132, 2008.

[10] G. Falqui, "On a Camassa-Holm type equation with two dependent variables," Journal of Physics A, vol. 39, no. 2, pp. 327342, 2006.

[11] J. Escher, O. Lechtenfeld, and Z. Yin, "Well-posedness and blowup phenomena for the 2-component Camassa-Holm equation," Discrete and Continuous Dynamical Systems A, vol. 19, no. 3, pp. 493-513, 2007.

[12] C. Guan and Z. Yin, "Global existence and blow-up phenomena for an integrable two-component Camassa-Holm shallow water system," Journal of Differential Equations, vol. 248, no. 8, pp. 2003-2014, 2010

[13] C. Guan and Z. Yin, "Global weak solutions for a twocomponent Camassa-Holm shallow water system," Journal of Functional Analysis, vol. 260, no. 4, pp. 1132-1154, 2011.

[14] D. Henry, "Infinite propagation speed for a two component Camassa-Holm equation," Discrete and Continuous Dynamical Systems B, vol. 12, no. 3, pp. 597-606, 2009.

[15] G. Gui and Y. Liu, "On the Cauchy problem for the twocomponent Camassa-Holm system," Mathematische Zeitschrift, vol. 268, no. 1-2, pp. 45-66, 2011.
[16] O. G. Mustafa, "On smooth traveling waves of an integrable two-component Camassa-Holm shallow water system," Wave Motion, vol. 46, no. 6, pp. 397-402, 2009.

[17] Q. Hu and Z. Yin, "Well-posedness and blow-up phenomena for a periodic two-component Camassa-Holm equation," Proceedings of the Royal Society of Edinburgh A, vol. 141, no. 1, pp. 93-107, 2011.

[18] Q. Hu and Z. Yin, "Global existence and blow-up phenomena for a periodic 2-component Camassa-Holm equation," Monatshefte für Mathematik, vol. 165, no. 2, pp. 217-235, 2012.

[19] D. Holm, L. Ó. Náraigh, and C. Tronci, "Singular solutions of a modified two-component Camassa-Holm equation," Physical Review E, vol. 79, Article ID 016601, 2009.

[20] G. Lv and M. Wang, "Some remarks for a modified periodic Camassa-Holm system," Discrete and Continuous Dynamical Systems A, vol. 30, no. 4, pp. 1161-1180, 2011.

[21] Y. Fu, Y. Liu, and C. Qu, "Well-posedness and blow-up solution for a modified two-component periodic Camassa-Holm system with peakons," Mathematische Annalen, vol. 348, no. 2, pp. 415448, 2010.

[22] S. Yu, "Well-posedness and blow-up for a modified twocomponent Camassa-Holm equation," Applicable Analysis, vol. 91, no. 7, pp. 1321-1337, 2012.

[23] C. Guan, K. Karlsen, and Z. Yin, "Well-posedness and blowup phenomena for a modified two-component Camassa-Holm equation, Nonlinear partial differential equations and hyperbolic wave phenomena," in Contemporary Mathematics, vol. 526, pp. 199-220, American Mathematical Society, Providence, RI, USA, 2010.

[24] W. Tan and Z. Yin, "Global dissipative solutions of a modified two-component Camassa-Holm shallow water system," Journal of Mathematical Physics, vol. 52, no. 3, Article ID 033507, 2011.

[25] Z. Guo and L. Ni, "Persistence properties and unique continuation of solutions to a two-component Camassa-Holm equation," Mathematical Physics, Analysis and Geometry, vol. 14, no. 2, pp. 101-114, 2011.

[26] W. Tan and Z. Yin, "Global periodic conservative solutions of a periodic modified two-component Camassa-Holm equation," Journal of Functional Analysis, vol. 261, no. 5, pp. 1204-1226, 2011.

[27] Z. Guo, M. Zhu, and L. Ni, "Blow-up criteria of solutions to a modified two-component Camassa-Holm system," Nonlinear Analysis. Real World Applications, vol. 12, no. 6, pp. 3531-3540, 2011.

[28] Z. Guo and M. Zhu, "Wave breaking for a modified twocomponent Camassa-Holm system," Journal of Differential Equations, vol. 252, no. 3, pp. 2759-2770, 2012.

[29] L. Jin and Z. Guo, "A note on a modified two-component Camassa-Holm system," Nonlinear Analysis. Real World Applications, vol. 13, no. 2, pp. 887-892, 2012.

[30] W. Rui and Y. Long, "Integral bifurcation method together with a translation-dilation transformation for solving an integrable 2-component Camassa-Holm shallow water system," Journal of Applied Mathematics, vol. 2012, Article ID 736765, 21 pages, 2012.

[31] J. B. Li and Y. S. Li, "Bifurcations of travelling wave solutions for a two-component Camassa-Holm equation," Acta Mathematica Sinica, vol. 24, no. 8, pp. 1319-1330, 2008.

[32] J.-M. Ghidaglia, "Weakly damped forced Korteweg-de Vries equations behave as a finite-dimensional dynamical system in the long time," Journal of Differential Equations, vol. 74, no. 2, pp. 369-390, 1988. 
[33] Q. Hu and Z. Yin, "Blowup and blowup rate of solutions to a weakly dissipative periodic rod equation," Journal of Mathematical Physics, vol. 50, no. 8, Article ID 083503, 2009.

[34] Q. Hu, "Global existence and blow-up phenomena for a weakly dissipative 2-component Camassa-Holm system," Applicable Analysis, vol. 92, no. 2, pp. 398-410, 2013.

[35] Q. Hu, "Global existence and blow-up phenomena for a weakly dissipative periodic 2-component Camassa-Holm system," Journal of Mathematical Physics, vol. 52, no. 10, Article ID 103701, 2011.

[36] T. Kato, "Quasi-linear equations of evolution with application to partial differential equations," in Spectral Theory and Differential Equations, pp. 25-70, Springer, Berlin, Germany, 1975.

[37] A. Constantin and J. Escher, "Wave breaking for nonlinear nonlocal shallow water equations," Acta Mathematica, vol. 181, no. 2, pp. 229-243, 1998.

[38] L. Ni and Y. Zhou, "A new asymptotic behavior of solutions to the Camassa-Holm equation," Proceedings of the American Mathematical Society, vol. 140, no. 2, pp. 607-614, 2012.

[39] A. A. Himonas, G. Misiołek, G. Ponce, and Y. Zhou, "Persistence properties and unique continuation of solutions of the Camassa-Holm equation," Communications in Mathematical Physics, vol. 271, no. 2, pp. 511-522, 2007.

[40] Z. Guo, "Asymptotic profiles of solutions to the two-component Camassa-Holm system," Nonlinear Analysis. Theory, Methods \& Applications A, vol. 75, no. 1, pp. 1-6, 2012. 


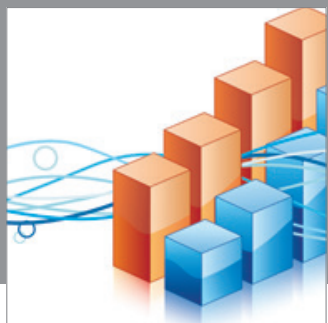

Advances in

Operations Research

mansans

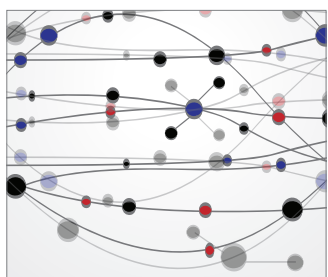

The Scientific World Journal
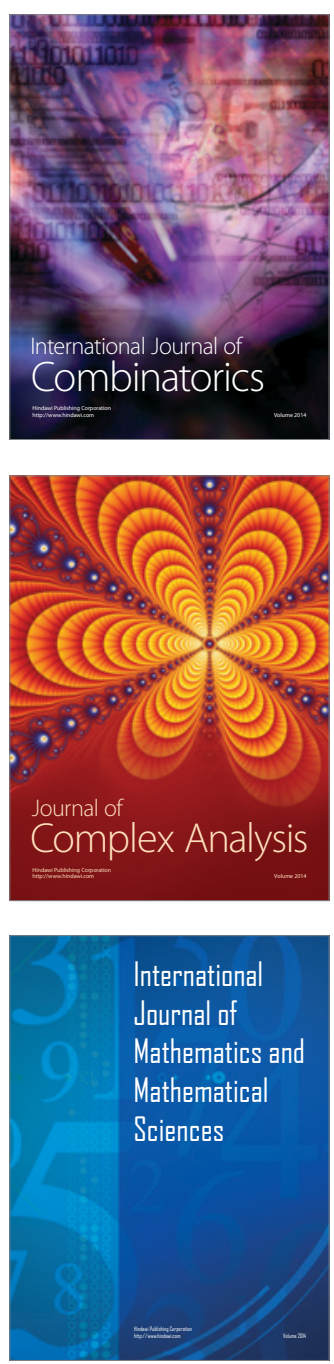
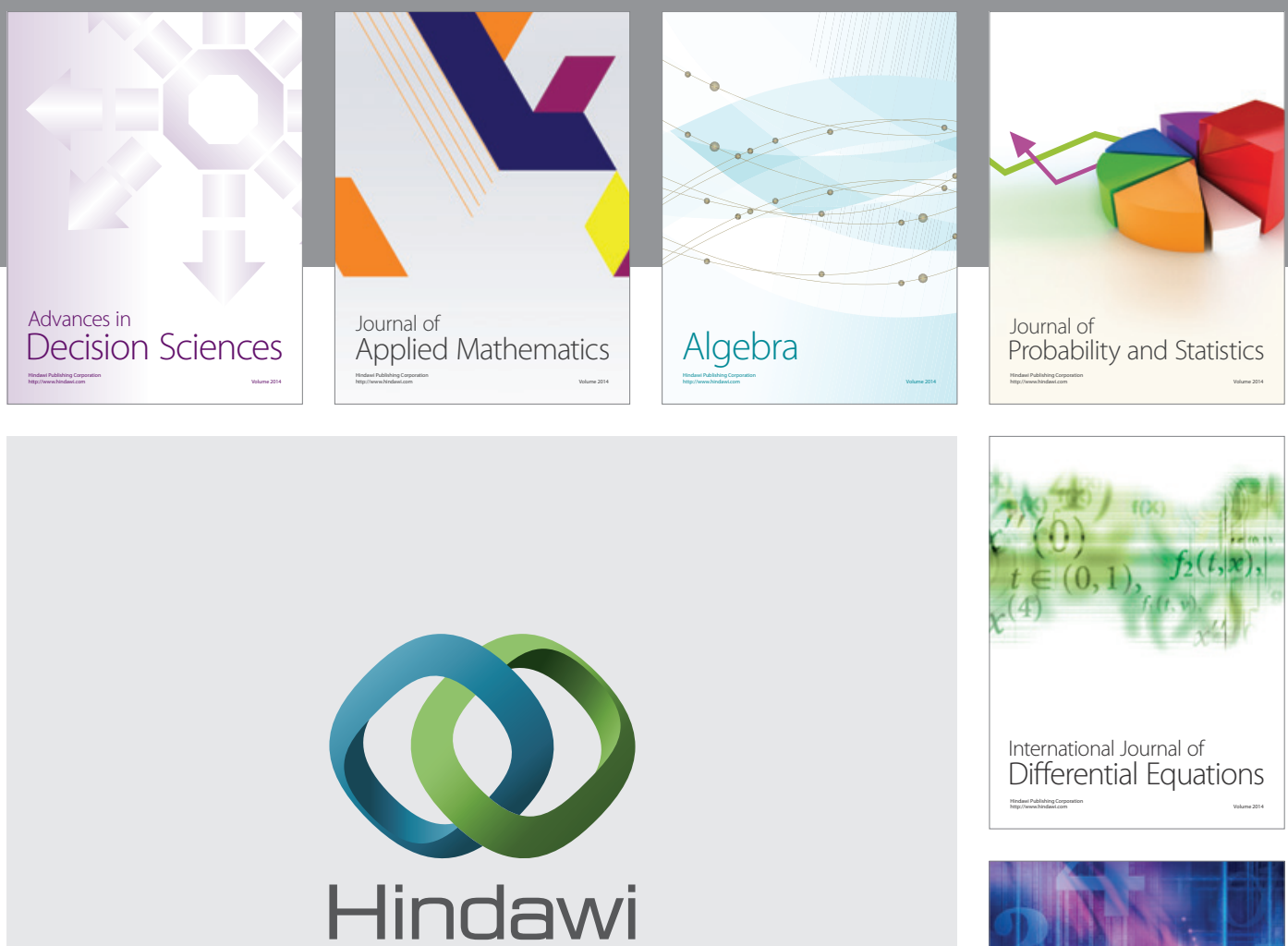

Submit your manuscripts at http://www.hindawi.com
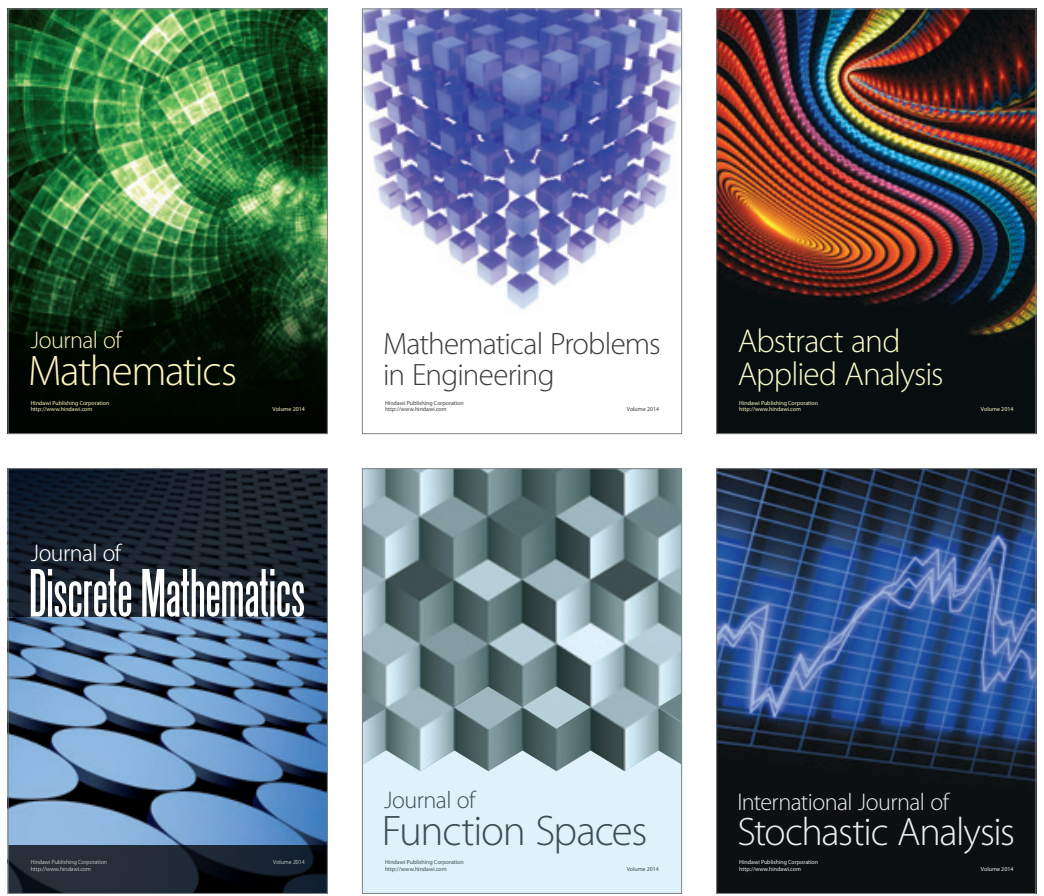

Journal of

Function Spaces

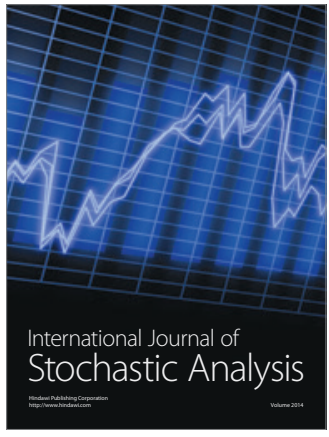

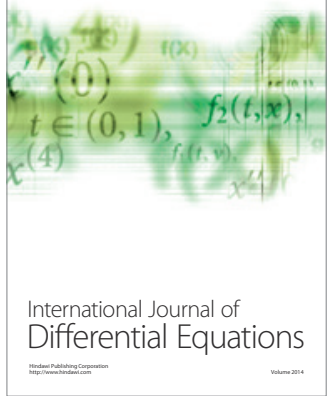
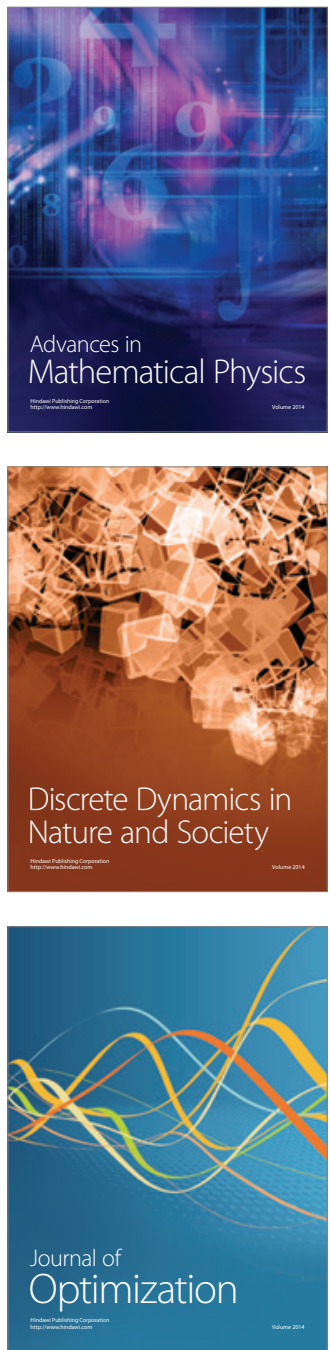